\title{
Ninjurin 2 rs118050317 gene polymorphism and endometrial cancer risk
}

\author{
Yimin Cheng ${ }^{1,2}$, Liting Yang ${ }^{1}$, Guangyao Shi ${ }^{1}$, Peng Chen ${ }^{1}$, Liang Li ${ }^{3}$, Hangrong Fang ${ }^{4}$ and Chao Chen ${ }^{1 *}$ (D)
}

\begin{abstract}
Background: Endometrial cancer is one of the most common female reproductive system tumors. Ninjurin2 (NINJ2) is a new adhesion factor. As a vascular susceptibility gene, it is highly expressed in other cancers and promotes the growth of cancer cells. We conducted an association analysis between NINJ2 gene polymorphism and endometrial cancer risk.

Methods: Five SNPs rs118050317, rs75750647, rs7307242, rs10849390 and rs 11610368 of NINJ2 gene were genotyped in 351 endometrial cancer patients and 344 healthy controls. The clinical index difference between cases and controls were tested by one-way analysis of variance. The allele and genotype frequency of cases and controls were been compared by Chi square test. The odds ratios (OR) with 95\% confidence interval (95\% Cl) were examined by logistic regression analysis.

Results: The SNP rs1 18050317 mutant allele $C$ and homozygote CC genotype were significant increased the endometrial cancer risk (OR 1.46, 95\% Cl 1.04-2.06, $p=0.028$; OR 8.43, 95\% Cl 1.05-67.89, $p=0.045$ ). In the clinical index analysis, there were significant higher quantities of CEA, CA125 and AFP in cases serum than controls.

Conclusion: The NINJ2 gene polymorphism loci rs 118050317 mutant allele C was associated with an increased risk of endometrial cancer. CEA, CA125 and AFP quantities were significant higher in endometrial cancer patients.
\end{abstract}

Keywords: Endometrial cancer, Ninjurin2, Clinical index, Single nucleotide polymorphisms

\section{Introduction}

Endometrial cancer is malignant tumor of endometrium epithelial, which also is one of the most common female reproductive system tumors [1]. In historically, endometrial cancer was been classified as type I (80-90\%) and type II (10-20\%), in histopathological classification divided tumors into endometrioid and non-endometrioid $[2,3]$. With the development of society and the improvement of economic conditions, the incidence of endometrial cancer has also increased year by year in China

\footnotetext{
*Correspondence: cchen898@nwu.edu.cn

${ }^{\dagger}$ Yimin Cheng and Liting Yang joint first authors.

${ }^{1}$ The National Engineering Research Centre for Miniaturized Detection Systems, College of Life Science, Northwest University, \#229 North TaiBai Road, Xi'an 710069, Shaanxi, China

Full list of author information is available at the end of the article
}

[4]. Increased estrogen exposure is a major risk factor for endometrial cancer, early menarche, infertility, obesity and late menopause are associated with endometrial cancer risk, too [5]. Moreover, women with a family history of endometrial cancer have a higher risk of the disease than those without a family history, suggesting that inherited genetic factors have an impact on the incidence of endometrial cancer [6].

In the genome-wide association study of endometrial cancer, the susceptibility locus close to $H N F 1 B$ on chromosome 17q was associated with endometrial cancer [7]. Vivo et al. replicated previously identified associations with genetic markers located at 17q12 (rs4430796) near the $H N F 1 B$ locus [8]. Five endometrial cancer risk SNPs loci on 13q22.1, 6q22.31, 8q24.21, 15q15.1 and 14q32.33 were identified by genome-wide association study $[9,10]$. Moreover, the SNP locus in ADIPOQ, MDM2, TP53 and 
leptin gene were associated with endometrial cancer, too $[5,11,12]$, respectively. Despite there were a lot of endometrial cancer studies, the endometrium carcinogenesis remains poorly understood.

Ninjurin2 (NINJ2) is a transmembrane protein that mediates cell-to-cell and cell-to-extracellular matrix interactions during development, differentiation, and regeneration of nervous system, the genetic polymorphisms of NINJ2 were associated with a decreased risk of Alzheimer's disease [13], ischemic stroke [14] and large artery atherosclerotic stroke [15]. Although there were significant association between NINJ2 gene polymorphisms and nervous system disease, but few studies were conducted between NINJ2 gene polymorphisms and cancer (Fig. 1).

The chronic inflammation may play an important role in endometrial carcinogenesis. IL- 6 gene had an association with endometrial cancer risk [16]. NINJ2 can regulate the expression of IL-6 in human vascular endothelial cells. In this study we investigated whether alterations in the NINJ2 gene can influence the risk of endometrial cancer. We performed the case control study to investigate the relationship between five SNPs (rs118050317, rs75750647, rs7307242, rs10849390 and rs11610368) of NINJ2 gene and endometrial cancer risk in Chinese women. We take A as the allele of mutant, then, the dominant model is: AA + AG compared with GG. The recessive model was: AA compared with AG + GG. The co-dominant model was: AA, AG and GG were compared. The additive model is: AA ratio GG. We used these four genetic models to study the relationship between SNPs of different genotypes and clinical indicators in patients with endometrial cancer.
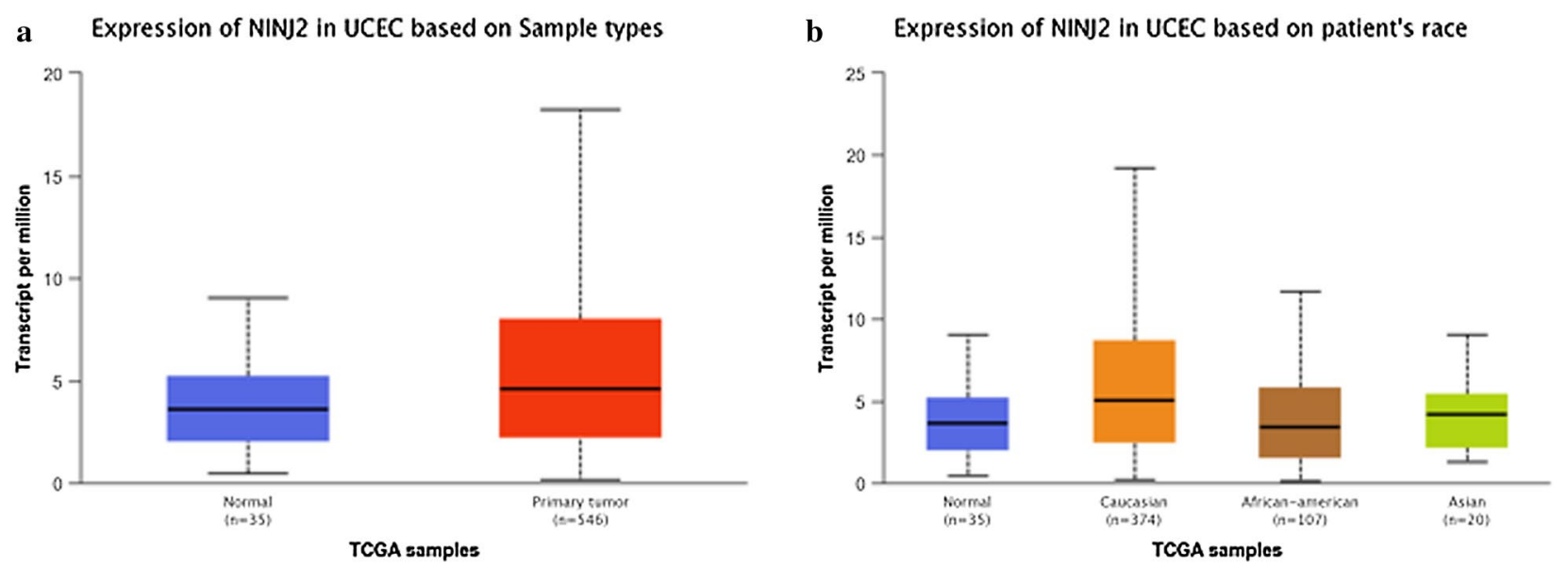

c

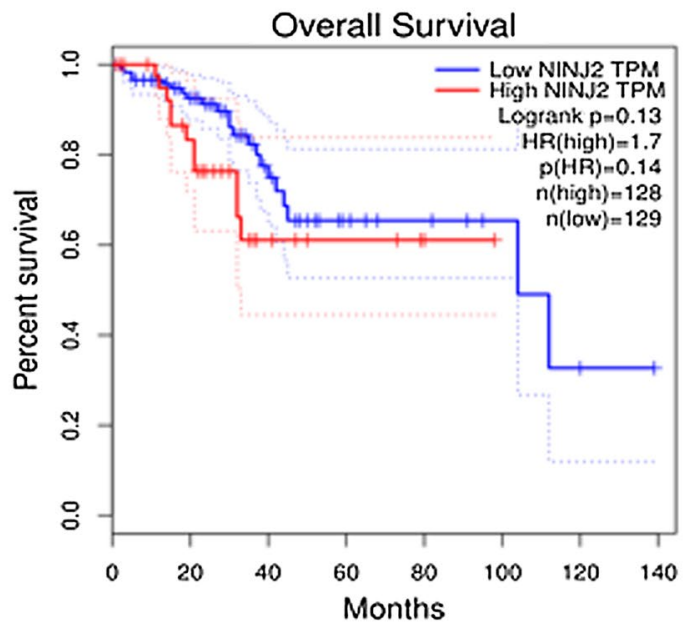

d

\begin{tabular}{|c|c|}
\hline Comparison & Statistical significance \\
\hline Normal-vs-Caucasian & $2.373699999889134 \mathrm{E}-07$ \\
\hline Normal-vS-AfricanAmerican & $3.341000 \mathrm{E}-01$ \\
\hline Normal-vs-Asian & $2.634800 \mathrm{E}-01$ \\
\hline Caucasian-vs-AfricanAmerican & $1.65445999999726 \mathrm{E}-05$ \\
\hline Caucasian-vs-Asian & $6.368600 \mathrm{E}-01$ \\
\hline AfricanAmerican-vs-Asian & $4.013800 \mathrm{E}-01$ \\
\hline
\end{tabular}

Fig. 1 Expression of NINJ2 in endometrial carcinoma. a The UALCAN database predicts the expression of NINJ2 in normal and cancer populations. b The UALCAN database predicts the expression of NINJ2 in patient's race. c The GEPIA database predicts the prognosis of NINJ2 in patients. $\mathbf{d}$ The $P$ value of the box diagram in Figure $B$ 


\section{Materials and methods Study population}

351 endometrial cancer patients $(55.66 \pm 8.46)$ were diagnosed and selected from Shaanxi Provincial Tumor Hospital. All patients were clinical diagnosed as endometrial cancer by pathology. All cases have no radiotherapy, chemotherapy and no other cancer history. Among them, 184 were smaller than 55 years old and 167 were greater than 55 years old. 344 healthy controls $(55.60 \pm 8.43)$ were selected from the physical examination center of Tang du physical examination center. All controls have no history of cancer and age matched with cases. Among them, 178 were smaller than 55 years old and 166 were greater than 55 years old (Table 1 ).

We collected clinical indicators of participants as much as possible, including Carcinoembryonic antigen (CEA), Carbohydrate antigen 125 (CA125), CA199, Human epididymis protein 4 (HE4), Alpha fetoprotein (AFP), Serum ferritin (SF) and Tumor necrosis factor (TNF). These clinical indicators are female tumor markers, and

Table 1 The basic information of cases and controls

\begin{tabular}{|c|c|c|c|}
\hline Variable & Cases $(n=351)$ & Controls $(n=344)$ & $p$ \\
\hline Age (Mean $\pm S D)$ & $55.66 \pm 8.46$ & $55.60 \pm 8.43$ & 0.923 \\
\hline$\leq 55$ & 184 & 178 & \\
\hline$>55$ & 167 & 166 & \\
\hline \multicolumn{4}{|l|}{ Clinical index } \\
\hline CEA & 305 & 105 & \\
\hline $\begin{array}{l}\text { Quantity in } \\
\text { serum(ng/ml) }\end{array}$ & $12.55 \pm 3.95$ & $2.24 \pm 3.14$ & $<0.001^{*}$ \\
\hline CA125 & 299 & 105 & \\
\hline $\begin{array}{l}\text { Quantity in serum } \\
(\mathrm{U} / \mathrm{ml})\end{array}$ & $24.23 \pm 56.30$ & $13.25 \pm 11.52$ & $0.048^{*}$ \\
\hline CA199 & 285 & 95 & \\
\hline $\begin{array}{l}\text { Quantity in serum } \\
(\mathrm{U} / \mathrm{ml})\end{array}$ & $21.21 \pm 58.23$ & $13.91 \pm 11.62$ & 0.226 \\
\hline AFP & 303 & 105 & \\
\hline $\begin{array}{l}\text { Quantity in serum } \\
(\mathrm{ng} / \mathrm{ml})\end{array}$ & $9.75 \pm 5.28$ & $2.77 \pm 0.97$ & $<0.001^{*}$ \\
\hline
\end{tabular}

CEA carcinoembryonic antigen, $C A$ carbohydrate antigen, AFP alpha fetoprotein ${ }^{*} p<0.05$ indicates statistical significance their dynamic changes are related to the disease, which can provide an important basis for the judgment, prognosis and treatment of the disease [17, 18]. All participators provided informed consent and the Ethical Committee approved by the study.

\section{SNP selection and genotyping}

Five SNPs rs118050317, rs75750647, rs7307242, rs10849390 and rs11610368 were selected from NINJ2 gene based on the minor allele frequency was greater than 0.05 in global population. The whole genome DNA was extracted from $5 \mathrm{ml}$ peripheral blood using GoldMag-Mini whole blood genomic DNA purification kit (GoldMag Co. Ltd. Xi'an City, China). The primers of genotyping were designed by Agena on-line software (https://agenacx.com/online-tools/), the primer of this study were list in Table 2. Agena MassARRAY platform was used to detect the five SNPs in case and control group (Agena Bioscience, SanDiego, CA, USA).

\section{Statistical analysis}

The clinical index quantity in serum difference was tested by one-way analysis of variance by SPSS16.0 [19], see the following formula: (1) Establish test hypothesis and determine test level: H0: There was no difference between the two groups; H1: There are differences between the two groups, or they are not all equal; (2) Calculate the value of statistic F: $\mathrm{SS}_{1}$ is the mean sum of squares between the groups; $\mathrm{SS}_{2}$ is the sum of mean squares within the group. $\mathrm{N}$ is the number of groups. $\mathrm{MS}_{1}$ is between the groups; $\mathrm{MS}_{2}$ is within the group.

$$
\begin{aligned}
& \mathrm{MS}_{1}=\mathrm{SS}_{1} / \mathrm{N}-1 \\
& \mathrm{MS}_{2}=\mathrm{SS}_{2} / \text { DATA }-\mathrm{N} \\
& \mathrm{F}=\mathrm{MS}_{1} / \mathrm{MS}_{2}
\end{aligned}
$$

(3) Determine P value and make statistical inference. The Hardy-Weinberg equilibrium (HWE) $p$ value of each SNP in controls was calculated by exact test, $p$ value greater than 0.05 means the sample have reached

Table 2 Primers used in this study

\begin{tabular}{llll}
\hline SNPs & 2nd-PCRP & 1st-PCRP & UEP_SEQ \\
\hline rs118050317 & ACGTTGGATGGTGTAGTGATTGACACCTG & ACGTTGGATGACAGGAGCTGGTCATGTTGC & ggggtGCCGATGGGGAAGGATTAG \\
rs75750647 & ACGTTGGATGTGTTCGCTGTGTACTGGATG & ACGTTGGATGCCCCCACAAAATTACAAACC & CTAAAGCAGGGTGGA \\
rs7307242 & ACGTTGGATGAAATGCTTCTCCTGGAAGTC & ACGTTGGATGGCCCTAGCCTGTTTCTTAA & CCAGATCACTAGCTCTGA \\
rs10849390 & ACGTTGGATGTCACACAATCTCACAGGGAC & ACGTTGGATGGAAATCAGTACTGCCTGTGC & tcgCCCAGGGACAGCCCGCTGCC \\
rs11610368 & ACGTTGGATGTCTGTGACTCCTTGCCAATG & ACGTTGGATGCTCTGCAATGTTACACAGCC & CCTTGCCAATGGATAGAATAGAA \\
\hline
\end{tabular}

UEP SEQ unextended mini-sequencing primer 
a genetic balance. The formula of Fisher's Exact test is as follows: $p=(a+b) !(c+d) !(a+c) !(b+d) ! /(a ! b ! c ! d ! n !) . a, b$, $c, d$, are the data in the contingency table, respectively.

The allele and genotype distribution in cases and controls were compared by Chi square test. The odds ratios (OR) with 95\% confidence interval (95\% CI) were examined by logistic regression analysis before and after adjustment for age [20]. Suppose the dependent variable $Z$ and a set of independent variables $x 1, x 2, x 3, \ldots$, $\mathrm{xn}$, where $\mathrm{Z}$ is a continuous variable, we can fit a linear equation:

$$
\begin{aligned}
& \mathrm{p}(\mathrm{y}=1)=\mathrm{ez} /(1+\mathrm{ez}), \\
& \mathrm{p}(\mathrm{y}=0)=1 /(1+\mathrm{ez}), \mathrm{Z}=\beta 0+\beta 1 * \mathrm{x} 1 \\
& +\beta 2 * \mathrm{x} 2+\beta 3 * \mathrm{x} 3+\cdots+\beta \mathrm{n} * \mathrm{xn}
\end{aligned}
$$

The least square method is used to estimate the value of each $\beta$ coefficient.

odds $=\mathrm{p} /(1-\mathrm{p})$, OR: Event occurrence probability of the experimental group (odds1)/Occurrence rate of events in control group (odds2).

The linkage disequilibrium and haplotype construction were analyzed by Haploview v4.2 [21].

\section{Results}

In this study, we recruited 351 endometrial cancer patients and 344 healthy controls, the age of cases and controls were matched $(p=0.923)$. There were significant differences in CEA, CA125 and AFP quantity in serum between cases and controls $(p<0.001 ; p=0.048$; $p<0.001)$. All of three clinical indicators were higher quantity in cases than controls (Table 1).

In Table 3, we listed the basic information of the SNPs which including the chromosome, position, minor and common allele, the HWE $p$ value of all SNPs were greater than 0.05 , we calculated the minor allele frequency (MAF) of cases and controls. We found compared with common $\mathrm{G}$ allele, the rs118050317 $\mathrm{C}$ allele were significant increased 0.46 -fold endometrial cancer risk (OR 1.46, 95\% CI 1.04-2.06, $p=0.028)$.

In Table 4, we calculated the genotype distribution in cases and controls under four different genotype model (co-dominant, dominant, recessive and log-additive) before and after adjustment the age. After adjusted the age, compared with GG genotype carriers, the rs118050317 CC genotype carriers were significant increased the endometrial cancer risk under co-dominant model (OR 8.43, 95\% CI 1.05-67.89, $p=0.045$ ), the results also significant under log-additive model (OR 1.47, 95\% CI 1.04-2.07, $p=0.029$ ) (Additional file 1: Table S2).

In the association analysis between different SNPs and different clinical index, we found there was significant difference between rs7307242 different genotype and CEA and AFP quantity $(p=0.021, p<0.001)$, genotype AA corresponding to the highest quantity, followed by TT and AT genotype. rs75750647 different genotypes were significant associated with CA125 and CA199 quantity $(p=0.032, p=0.033)$, the AA genotype carriers had the highest CA125 and CA199 quantity. SNP loci rs11610368 different genotypes were significant associated with $\mathrm{HE} 4$ quantity $(p=0.010)$, genotype AA corresponding to the highest quantity, followed by GG and AG (Table 5, Additional file 1: Table S1).

\section{Discussion}

In this study, we did an association study between NINJ2 gene polymorphism and endometrial cancer risk in 351 endometrial cancer patients and 344 healthy controls. We found the SNP rs118050317 mutant allele C and homozygote $\mathrm{CC}$ genotype were significant increased the endometrial cancer risk. In the clinical index analysis, there was significant higher quantity of CEA, CA125 and

\begin{tabular}{|c|c|c|c|c|c|c|c|c|c|c|}
\hline \multirow[t]{2}{*}{ SNPs } & \multirow[t]{2}{*}{ Chromosome } & \multirow[t]{2}{*}{ Gene } & \multirow[t]{2}{*}{ Position } & \multirow[t]{2}{*}{ Minor allele } & \multirow{2}{*}{$\begin{array}{l}\text { Common } \\
\text { allele }\end{array}$} & \multirow[t]{2}{*}{ HWE $p$ value } & \multicolumn{2}{|l|}{ MAF } & \multirow[t]{2}{*}{ OR $(95 \% \mathrm{Cl})$} & \multirow[t]{2}{*}{$p$} \\
\hline & & & & & & & Case & Control & & \\
\hline rs118050317 & 12 & NINJ2 & 634,980 & C & G & 0.336 & 0.129 & 0.092 & $1.46(1.04-2.06)$ & $0.028^{*}$ \\
\hline rs75750647 & 12 & NINJ2 & 638,831 & $A$ & G & 0.617 & 0.333 & 0.314 & $1.09(0.87-1.37)$ & 0.440 \\
\hline rs7307242 & 12 & NINJ2 & 641,529 & $A$ & $\mathrm{~T}$ & 0.197 & 0.138 & 0.149 & $0.92(0.68-1.24)$ & 0.576 \\
\hline rs10849390 & 12 & NINJ2 & 646,086 & G & A & 0.638 & 0.372 & 0.360 & $1.05(0.85-1.31)$ & 0.646 \\
\hline rs11610368 & 12 & NINJ2 & 662,624 & $A$ & G & 0.053 & 0.127 & 0.130 & $0.97(0.71-1.33)$ & 0.869 \\
\hline
\end{tabular}
AFP in cases than controls.

Table 3 Basic information of candidate SNPs in this study

SNP single nucleotide polymorphisms, HWE Hardy-Weinberg equilibrium, MAF minor allele frequency, OR odds ratio, 95\% CI 95\% confidential interval ${ }^{*} p<0.05$ indicates statistical significance 
Table 4 Genotype frequencies of the SNPs and their associations with risk of endometrial cancer

\begin{tabular}{|c|c|c|c|c|c|c|c|c|}
\hline \multirow[t]{2}{*}{ SNP } & \multirow[t]{2}{*}{ Model } & \multirow[t]{2}{*}{ Genotype } & \multirow[t]{2}{*}{ Case } & \multirow[t]{2}{*}{ Control } & \multicolumn{2}{|c|}{ Without adjustment } & \multicolumn{2}{|c|}{ With adjustment of age } \\
\hline & & & & & OR $(95 \% \mathrm{Cl})$ & $p$ & OR $(95 \% \mathrm{Cl})$ & $p$ \\
\hline \multirow[t]{8}{*}{ rs118050317 } & Co-dominant & GG & 268 & 282 & 1 & & 1 & \\
\hline & & $C G$ & 74 & 61 & $1.28(0.87-1.86)$ & 0.206 & $1.28(0.88-1.87)$ & 0.202 \\
\hline & & $\mathrm{CC}$ & 8 & 1 & $8.42(1.05-67.76)$ & $0.045^{*}$ & $8.43(1.05-67.89)$ & $0.045^{*}$ \\
\hline & Dominant & GG & 268 & 282 & 1 & & 1 & \\
\hline & & $C G+C C$ & 82 & 62 & $1.39(0.96-2.01)$ & 0.080 & $1.40(0.96-2.02)$ & 0.078 \\
\hline & Recessive & $G G+C G$ & 342 & 343 & 1 & & 1 & \\
\hline & & CC & 8 & 1 & $8.02(1.00-64.50)$ & 0.050 & $8.03(1.00-64.52)$ & 0.050 \\
\hline & Log-additive & - & - & - & $1.46(1.04-2.06)$ & $0.029^{*}$ & $1.47(1.04-2.07)$ & $0.029^{*}$ \\
\hline
\end{tabular}

OR odds ratio, $95 \% \mathrm{Cl} 95 \%$ confidential interval

${ }^{*} p<0.05$ indicates statistical significance

Table 5 The association between SNPs of NINJ2 and clinical index of endometrial cancer

\begin{tabular}{|c|c|c|c|c|c|}
\hline SNP & Clinical Index & Genotype & Number in cases & $\begin{array}{l}\text { Quantity in blood } \\
\text { (mean } \pm \text { SD) }\end{array}$ & $p$ \\
\hline \multirow[t]{6}{*}{ rs7307242 } & CEA (ng/ml) & AA & 7 & $14.94 \pm 3.97$ & $0.021^{*}$ \\
\hline & & AT & 69 & $11.56 \pm 3.55$ & \\
\hline & & $\mathrm{TT}$ & 229 & $12.78 \pm 4.01$ & \\
\hline & $\operatorname{AFP}(\mathrm{ng} / \mathrm{ml})$ & AA & 7 & $19.55 \pm 21.16$ & $<0.001^{*}$ \\
\hline & & AT & 69 & $9.23 \pm 3.82$ & \\
\hline & & $\mathrm{TT}$ & 227 & $9.61 \pm 4.23$ & \\
\hline \multirow[t]{6}{*}{ rs75750647 } & CA125 (U/ml) & AA & 35 & $46.51 \pm 131.23$ & $0.032^{*}$ \\
\hline & & $A G$ & 129 & $24.09 \pm 44.88$ & \\
\hline & & GG & 135 & $18.6 \pm 24.39$ & \\
\hline & CA199 (U/ml) & AA & 31 & $46.19 \pm 163.92$ & $0.033^{*}$ \\
\hline & & $A G$ & 124 & $15.9 \pm 14.45$ & \\
\hline & & GG & 130 & $20.32 \pm 28.95$ & \\
\hline \multirow[t]{3}{*}{ rs11610368 } & HE4 (pg/ml) & AA & 3 & $295.38 \pm 390.97$ & $0.010^{*}$ \\
\hline & & AG & 65 & $90.37 \pm 99.74$ & \\
\hline & & GG & 233 & $91.49 \pm 113.83$ & \\
\hline
\end{tabular}

CEA carcinoembryonic antigen, CA carbohydrate antigen, HE4 human epididymis protein 4, AFP alpha fetoprotein, $S F$ serum ferritin, TNF tumor necrosis factor ${ }^{*} p<0.05$ indicates statistical significance

The gene encodes NINJ2 is located on chromosome 12p13. Toshiyuki et al. showed NINJ2 is a cell surface adhesion molecule [22]. As a vascular susceptibility gene, the SNPs in NINJ2 were genotyped to test for the association between variants of NINJ2 and dementia risk. It was found that the homozygosity of two SNPs rs11833579 and rs12425791 were associated with a decreased risk of Alzheimer's disease [13]. Moreover, the researchers also found the SNP rs12425791 of NINJ2 was significantly associated with ischemic stroke, and A allele increases the susceptibility of stroke [23]. In a family-based case control study, the A allele of rs 11833579 may play a role in mediating susceptibility to ischemic stroke [14]. A functional polymorphism loci rs3809263 in the NINJ2 promoter was significant decreased the large artery atherosclerotic stroke risk. Moreover, the AA genotype carriers had significantly increased NINJ2 mRNA expression levels in Chinese population [15].

Previous study reported NINJ1, a homologue of NINJ2, can mediate inflammation processes [24]. In addition, NINJ2 is expressed in lymphocyte cell. Inflammation was likely to increase risk of developing endometrial cancer [25]. Wang et al. found NINJ2 can regulate the express of gene that associated with inflammation in human vascular endothelial cells, such as IL-6 [26]. In other study, a hypothesis about chronic inflammation may play an 
important role in endometrial carcinogenesis were investigated. The association between SNP in inflammatory pathway genes and endometrial cancer risk were conducted and found IL-6 SNP rs2069852 had an association with endometrial cancer risk [16]. So we speculate the rs118050317 C allele of NINJ2 through regulate the IL-6 expression to influence the ERK-NF- $\mathrm{kB}$ signaling pathway of endometrial cancer growth [27]. The underlying mechanism need to be further investigated.

In this study, there were some limitations. First, endometrial cancer is a high incidence of female reproductive system tumors but relatively low incidence in the population. So the example size of our study was relatively small, we will collect as many samples as we can and verify our results in a larger population in future. Second, the clinical information were limited in this study, to enrich our study, we would continue to collect the information of patients and prognosis information to analyze the association between NINJ2 gene and clinical information. The underlying mechanism of NINJ2 gene involving in endometrial cancer need to be researched next step.

\section{Conclusion}

In conclusion, the NINJ2 gene polymorphism loci rs118050317 mutant allele $\mathrm{C}$ was associated with an increased risk of endometrial cancer and there was significant higher quantity in serum of CEA, CA125 and AFP in cases than controls.

\section{Supplementary information}

Supplementary information accompanies this paper at https://doi. org/10.1186/s12935-020-01646-5.

Additional file 1: Table S1. The association between SNPS of NINJ2 and clinical index of endometrial cancer. Additional file 1: Table S2. Genotype frequencies of the SNPs and their associations with risk of endometrial cancer

\section{Abbreviations}

NINJ2: Ninjurin2; CEA: Carcinoembryonic antigen; CA125: Carbohydrate antigen 125; AFP: Alpha fetoprotein; HE4: Human epididymis protein 4; SF: Serum ferritin; TNF: Tumor necrosis factor; MAF: minor allele frequency; OR: Odds ratios; 95\% Cl: 95\% confidence interval; HWE: Hardy-weinberg equilibrium; SNP: Single nucleotide polymorphism; ERK: Extracellular signal-regulated kinase.

\section{Acknowledgements}

We would like to thank all the patients and individuals in this study for their participation. We are also very grateful for the assistance of clinicians and all hospital staff of the Shaanxi Provincial Cancer Hospital and Tang du physical examination center who contributed blood samples and data for this study.

\section{Authors' contributions}

PC and CC conceived and designed the project. YMC and LL recruited and collected study samples. LTY selected the SNPS and designed primers. YMC and HRF performed the experiments. GYS performed and analyzed the data. YMC and LTY wrote and revised the manuscript.

\section{Funding}

This study was funded through Scientific Research Program of Shaanxi Education Department in 2018 (NO.18JK0624).

\section{Availability of data and materials}

All the data supporting the findings of this study are contained in the manuscript.

\section{Ethics approval and consent to participate}

The use of human blood sample and the protocol in this study were strictly comply with the criterions of the Declaration of Helsinki and were approved by the Ethics Committee of Shaanxi Provincial Tumor Hospital and Tang du physical examination center. Written informed consent was received from each participant.

\section{Consent for publication}

Not applicable.

\section{Competing interests}

The authors declare that they have no competing interest.

\section{Author details}

${ }^{1}$ The National Engineering Research Centre for Miniaturized Detection Systems, College of Life Science, Northwest University, \#229 North TaiBai Road, Xi'an 710069, Shaanxi, China. ${ }^{2}$ The Hospital of Xi'an Shiyou University, Xi'an, People's Republic of China. ${ }^{3}$ Department of Radiotherapy, Shaanxi Provincial Tumor Hospital, Xi'an, People's Republic of China. ${ }^{4}$ Department of Pathology, Xi'an No.3 Hospital, The Affiliated Hospital of Northwest University, Xi'an, People's Republic of China.

Received: 9 June 2020 Accepted: 9 November 2020

Published online: 04 January 2021

\section{References}

1. Amant F, Moerman P, Neven P, Timmerman D, Van Limbergen E, Vergote I. Endometrial cancer. Lancet (Lond Engl). 2005;366(9484):491-505.

2. Colombo N, Creutzberg C, Amant F, Bosse T, Gonzalez-Martin A, Ledermann J, Marth C, Nout R, Querleu D, Mirza MR, et al. ESMO-ESGO-ESTRO consensus conference on endometrial cancer: diagnosis, treatment and follow-up. Int J Gynecol Cancer. 2016;26(1):2-30.

3. Lu Z, Chen J. Introduction of WHO classification of tumours of female reproductive organs, fourth edition. Zhonghua Bing li Xue Za Zhi Chin J Pathol. 2014;43(10):649-50.

4. Gao Y, Zhao M, Dai X, Tong M, Wei J, Chen Q. The prevalence of endometrial cancer in pre- and postmenopausal Chinese women. Menopause (NY, NY). 2016;23(8):884-7.

5. Yoneda T, Kuboyama A, Kato K, Ohgami T, Okamoto K, Saito T, Wake N. Association of MDM2 SNP309 and TP53 Arg72Pro polymorphisms with risk of endometrial cancer. Oncol Rep. 2013;30(1):25-34.

6. Win AK, Reece JC, Ryan S. Family history and risk of endometrial cancer: a systematic review and meta-analysis. Obstet Gynecol. 2015;125(1):89-98.

7. Spurdle AB, Thompson DJ, Ahmed S, Ferguson K, Healey CS, O'Mara T, Walker LC, Montgomery SB, Dermitzakis ET, Fahey P, et al. Genome-wide association study identifies a common variant associated with risk of endometrial cancer. Nat Genet. 2011;43(5):451-4.

8. De Vivo I, Prescott J, Setiawan VW, Olson SH, Wentzensen N, Attia J, Black A, Brinton L, Chen C, Chen C, et al. Genome-wide association study of endometrial cancer in E2C2. Hum Genet. 2014;133(2):211-24.

9. Cheng TH, Thompson DJ, O'Mara TA, Painter JN, Glubb DM, Flach S, Lewis A, French JD, Freeman-Mills L, Church D, et al. Five endometrial cancer risk loci identified through genome-wide association analysis. Nat Genet. 2016;48(6):667-74.

10. Painter JN, Kaufmann S, O'Mara TA, Hillman KM, Sivakumaran H, Darabi H, Cheng THT, Pearson J, Kazakoff S, Waddell N, et al. A common variant at the 14q32 endometrial cancer risk locus activates AKT1 through YY1 binding. Am J Hum Genet. 2016;98(6):1159-69.

11. Bienkiewicz J, Smolarz B, Malinowski A. Association between single nucleotide polymorphism +276G > T (rs1501299) in ADIPOQ and endometrial cancer. Pathol Oncol Res POR. 2016;22(1):135-8. 
12. Bienkiewicz J, Romanowicz H, Malinowski A, Smolarz B. Association of single nucleotide polymorphism -2548 G/A (rs12112075) of leptin gene with endometrial cancer and uterine leiomyomas. Eur J Obstet Gynecol Reprod Biol. 2017;218:113-8.

13. Lin KP, Chen SY, Lai LC, Huang YL, Chen JH, Chen TF, Sun Y, Wen LL, Yip PK, Chu YM, et al. Genetic polymorphisms of a novel vascular susceptibility gene, Ninjurin2 (NINJ2), are associated with a decreased risk of Alzheimer's disease. PLoS One. 2011;6(6):e20573.

14. Zhu Y, Liu K, Tang X, Wang J, Yu Z, Wu Y, Chen D, Wang X, Fang K, Li N, et al. Association between NINJ2 gene polymorphisms and ischemic stroke: a family-based case-control study. J Thromb Thrombolysis. 2014;38(4):470-6.

15. Zhang Z, Ni G, Xu G, Xu J, Liu X. A novel functional polymorphism in the NINJ2 promoter predicts risk of large artery atherosclerotic stroke. Mol Neurobiol. 2016;53(10):7178-83.

16. Delahanty RJ, Xiang YB, Spurdle A, Beeghly-Fadiel A, Long J, Thompson D Tomlinson I, Yu H, Lambrechts D, Dork T, et al. Polymorphisms in inflammation pathway genes and endometrial cancer risk. Cancer Epidemiol Biomark Prev Publ Am Assoc Cancer Res Cospons Am Soc Prev Oncol. 2013;22(2):216-23.

17. Shi C, van der Wal HH, Silljé HHW, Dokter MM, van den Berg F, Huizinga $L$, Vriesema M, Post J, Anker SD, Cleland JG, et al. Tumour biomarkers: association with heart failure outcomes. J Intern Med. 2020;288(2):207-18.

18. Samy N, Ragab HM, El Maksoud NA, Shaalan M. Prognostic significance of serum Her2/neu, BCL2, CA15-3 and CEA in breast cancer patients: a short follow-up. Cancer Biomark Sect A Dis Mark. 2010;6(2):63-72.

19. Li S, Jin T, Zhang J, Lou H, Yang B, Li Y, Chen C, Zhang Y. Polymorphisms of TREH, IL4R and CCDC26 genes associated with risk of glioma. Cancer Epidemiol. 2012;36(3):283-7.

20. Yuan LJ, Jin TB, Yin JK, Du XL, Wang Q, Dong R, Wang SZ, Cui Y, Chen C, Lu JG. Polymorphisms of tumor-related genes IL-10, PSCA, MTRR and NOC3L are associated with the risk of gastric cancer in the Chinese Han population. Cancer Epidemiol. 2012;36(6):e366-72.
21. Li G, Bai M, Guo C, Zhu L, Wang L, Yuan D, Jin T, He Y. Association analysis of CHRNA3 polymorphisms with schizophrenia in a Chinese Han population: a case-control study. Medicine. 2018;97(23):e10863.

22. Araki T, Milbrandt J. Ninjurin2, a novel homophilic adhesion molecule, is expressed in mature sensory and enteric neurons and promotes neurite outgrowth. J Neurosci. 2000;20(1):187-95.

23. Wang X, Zhang J, Liu Y, Zhang Y. Relationship between nerve injuryinduced protein gene 2 polymorphism and stroke in Chinese Han population. J Biomed Res. 2011;25(4):287-91.

24. Ahn BJ, Lee HJ, Shin MW, Choi JH, Jeong JW, Kim KW. Ninjurin1 is expressed in myeloid cells and mediates endothelium adhesion in the brains of EAE rats. Biochem Biophys Res Commun. 2009;387(2):321-5.

25. Trabert B, Eldridge RC, Pfeiffer RM, Shiels MS, Kemp TJ, Guillemette C, Hartge P, Sherman ME, Brinton LA, Black A, et al. Prediagnostic circulating inflammation markers and endometrial cancer risk in the prostate, lung, colorectal and ovarian cancer (PLCO) screening trial. Int J Cancer. 2017;140(3):600-10.

26. Wang J, Fa J, Wang P, Jia X, Peng H, Chen J, Wang Y, Wang C, Chen Q, Tu X, et al. NINJ2-A novel regulator of endothelial inflammation and activation. Cell Signal. 2017;35:231-41.

27. Che Q, Liu BY, Wang FY, He YY, Lu W, Liao Y, Gu W, Wan XP. Interleukin 6 promotes endometrial cancer growth through an autocrine feedback loop involving ERK-NF-kappaB signaling pathway. Biochem Biophys Res Commun. 2014;446(1):167-72.

\section{Publisher's Note}

Springer Nature remains neutral with regard to jurisdictional claims in published maps and institutional affiliations.
Ready to submit your research? Choose BMC and benefit from:

- fast, convenient online submission

- thorough peer review by experienced researchers in your field

- rapid publication on acceptance

- support for research data, including large and complex data types

- gold Open Access which fosters wider collaboration and increased citations

- maximum visibility for your research: over $100 \mathrm{M}$ website views per year

At BMC, research is always in progress.

Learn more biomedcentral.com/submissions 